\title{
The Nature of the Insensitivity of Gram-Negative Bacteria towards Penicillins
}

\author{
By R. SUTHERLAND \\ Beecham Research Laboratories Limited, Research Division, Betchrvorth, Surrey
}

(Received 1 July 1963)

\section{SUMMARY}

The insensitivity of Gram-negative bacteria towards different penicillins has been correlated with the inactivation of these compounds by the penicillinases produced by these bacteria. Penicillins such as phenethicillin, propicillin, methicillin, and cloxacillin were relatively inactive against Gram-negative bacteria, but resistance was not accompanied by inactivation of these compounds. With ampicillin and benzylpenicillin, however, bacterial resistance was associated with destruction of the penicillins. Ampicillin was more stable to the penicillinases produced by certain Gram-negative bacteria than was benzylpenicillin and was correspondingly more active against these organisms. Gram-negative bacteria which produced little or no penicillinase were two to four times more sensitive to ampicillin than to benzylpenicillin, but ampicillin was at least ten times more active than benzylpenicillin against several penicillinaseproducing coliform organisms. All strains of bacteria which were resistant to both ampicillin and benzylpenicillin were capable of inactivating both compounds, but the insensitivity of these bacteria was not necessarily due solely to penicillinase production. Strains of Pseudomonas aeruginosa and certain strains of Escherichia coli and Proteus mirabilis were able to grow in high concentrations of these antibiotics, whereas with the strains of Aerobacter aerogenes, Proteus morganii and Proteus vulgaris examined, the resistance appeared to be due solely to inactivation of the penicillins.

\section{INTRODUCTION}

The destruction of benzylpenicillin by certain Gram-negative bacteria was reported first by Abraham \& Chain (1940) and, later, by Bondi \& Dietz (1944), but both groups of workers also observed that the resistance of bacteria to penicillins was not invariably associated with penicillinase formation. Similar findings with ampicillin were reported by Rolinson \& Stevens (1961) who showed that certain Gram-negative bacteria were capable of destroying the antibiotic, but that others were capable of growing in high concentrations of it; these results have been confirmed by other workers (Stewart, Coles, Nixon \& Holt, 1961; Trafford et al. 1962; Ayliffe, 1963). Thus, with ampicillin, as with benzylpenicillin, penicillinase appeared to occupy an indeterminate role in the resistance of Gram-negative bacteria towards penicillins. More recently, the characteristics of the penicillinases elaborated by certain Gram-negative bacteria have been investigated by various workers who have reported striking differences in the activities of these enzymes against various penicillins. For example, Auhagen et al. (1962) and Smith (1963) described strains of Escherichia coli which destroyed benzylpenicillin but which 
had little or no effect on ampicillin, propicillin, methicillin or oxacillin. Ayliffe (1963) showed that ampicillin was more stable than benzylpenicillin to the penicillinase produced by a strain of $\boldsymbol{E}$. coli, but, in addition, reported that ampicillin was inactivated as rapidly, or more rapidly, by penicillinases produced by strains of Proteus and Klebsiella. In these laboratories, Mr F. R. Batchelor (personal communication) has measured marked variation in the reaction of several penicillins to the penicillinases produced by a number of Gram-negative bacteria. It seemed possible that in some cases the differences in the sensitivities of some strains of Gram-negative bacteria towards various penicillins might be correlated with the relative stabilities of these compounds to the bacterial penicillinases. The results reported here were derived from experiments designed to investigate the relationship between the penicillinase activity of Gram-negative bacteria and the sensitivity of these organisms to penicillins, with a view to assessing the significance of these enzymes as factors in the resistance of bacteria to these antibiotics. The experiments comprised tests for antibacterial activity, measurement of the destruction of penicillins by bacteria, and observation of the growth of bacteria in the presence of penicillins. In addition, certain tests were also carried out to distinguish between penicillin inactivation by $\beta$-lactamases (penicillinases), which yield penicilloic acids, and deacylases (penicillin amidases) which yield 6-amino-penicillanic acid (6-APA).

\section{METHODS}

Measurement of the antibacterial activities of penicillins. Minimal inhibitory concentrations of various penicillins against bacteria were determined by twofold serial dilution in nutrient broth (Oxoid No. 2). The antibiotics were incorporated in graded concentrations in $5 \mathrm{ml}$. volumes of nutrient broth which were inoculated with 1 drop $(0.03 \mathrm{ml}$.) of an overnight culture of the test organism. Inhibitory concentrations were measured after overnight incubation at $37^{\circ}$. In other experiments, the effect on the antibacterial activity of the penicillins of altering the inoculum size was measured.

Measurement of penicillin destruction. The destruction of penicillins incubated overnight in the antibacterial tests described above was estimated by microbiological assay of residual penicillin activity. Samples were taken from the cultures and were plated in punch-holes in large rectangular plates containing nutrient agar seeded with Bacillus subtilis (ATCC 6633). In these assays, standard lines were prepared from standard solutions of penicillins which had been incubated overnight at $37^{\circ}$ in nutrient broth to compensate for thermal inactivation of penicillin. The plates containing test and standard samples were incubated overnight at $29^{\circ}$ when the diameters of zones of inhibition were measured in the usual way. Tests were also carried out to estimate the extent of penicillin inactivation due to the experimental procedure adopted, and which represented destruction additional to that occurring in the antibacterial tests. Likely factors appeared to be change in $\mathrm{pH}$ value of cultures, and the penicillinase activities of test cultures during the microbiological assay procedures.

Measurement of the effect of penicillins on the growth of bacteria. Optically matched tubes containing selected concentrations of penicillins in $10 \mathrm{ml}$. volumes of nutrient broth were incubated with $0.5 \mathrm{ml}$. or $1.0 \mathrm{ml}$. of an overnight culture of test organism. 


\section{Penicillin insensitivity}

The solutions were incubated at $37^{\circ}$ in a water bath, and growth was measured turbidimetrically over a period of $24 \mathrm{hr}$ by means of an EEL nephelometer. In addition, a replicate set of cultures was prepared in an identical fashion and samples were taken from these at regular intervals and assayed for penicillin content as described above.

Quantitative estimation of penicillins by chromatography. Samples were taken from broth cultures of Pseudomonas aeruginosa which had been incubated overnight at $37^{\circ}$ with known concentrations of ampicillin or benzylpenicillin and these were assayed as follows. One drop (about $6 \mu \mathrm{l}$.) was placed on the origin of filter-paper tapes (1 cm. wide, Whatman No. 1) and developed by descending chromatography in a butanol + pyridine + water $(1+1+1$, by vol.) system. Tapes spotted with nutrient broths containing from 2.5 to $100 \mu \mathrm{g}$. ampicillin or benzylpenicillin $/ \mathrm{ml}$. were treated in a like manner. After $16 \mathrm{hr}$, the tapes were placed on the surface of large rectangular assay plates containing nutrient agar seeded with Bacillus subtilis (ATCC 6633) and incubated for $24 \mathrm{hr}$ at $29^{\circ}$. The penicillin concentrations of the test samples were estimated from standard lines derived from standard samples.

Chromatographic estimation of 6-aminopenicillanic acid. Samples were taken from the antibacterial tests and treated as above for quantitative estimation of penicillin by chromatography. The tapes were sprayed with $5 \%$ sodium bicarbonate solution and $5 \%$ phenylacetyl chloride in acetone before being plated for microbiological assay (Batchelor, Doyle, Nayler \& Rolinson, 1959).

Chromatographic examination for penicilloic acid. Samples from antibacterial tests with ampicillin and benzylpenicillin were spotted on large sheets (Whatman paper No. 1) and developed in the butanol + pyridine + water system as described above. The chromatograms were sprayed with the starch-iodine reagent of Thomas (1961), and the zones which developed compared with those produced by the penicilloic acids which had been prepared by treatment of standard solutions of ampicillin and benzylpenicillin with penicillinase.

\section{RESULTS}

\section{Preliminary}

Destruction of penicillin due to change in $\mathrm{pH}$ values of bacterial cultures was considered to be negligible as a result of experiments which showed that there was little inactivation of standard solutions of benzylpenicillin incubated overnight in nutrient broth buffered at $\mathrm{pH}$ 5.0. This was the lowest $\mathrm{pH}$ value recorded for a number of overnight cultures of Enterobacteriaceae.

The amount of destruction of ampicillin or benzylpenicillin by bacterial penicillinase during the microbiological assay procedures was estimated by finding the effect of adding, at measured intervals, a culture of a penicillinase-producing strain of Staphylococcus aureus to standard solutions of these penicillins contained in punch-holes in an assay plate. There was significant inactivation only when the enzyme system was added within $30 \mathrm{~min}$. of placing the penicillin solution on the assay plate, owing to the very rapid diffusion of the penicillins into the surrounding medium. It seemed improbable therefore that there would be any marked destruction of penicillins during the microbiological assay as a result of the penicillinase produced by the Gram-negative bacteria being examined. 


\section{Examination for production of 6-aminopenicillanic acid (6-APA)}

Chromatographic examination of broth cultures in which there was marked destruction of penicillin as judged by microbiological assay, showed the presence of large amounts of the cognate penicilloic acid, and little or no 6-APA. The organisms tested included strains of Escherichia coli, Proteus, Pseudomonas aeruginosa and Aerobacter aerogenes. With one strain of Proteus rettgeri, however, an appreciable quantity of 6-APA was detected; the penicilloic acids of benzylpenicillin and 6-APA were also observed in cultures of this strain.

The activities of seven penicillins against Gram-negative bacteria

The relative activities of ampicillin, benzylpenicillin, phenoxymethylpenicillin, phenethicillin, propicillin, methicillin, and cloxacillin, against a variety of Gramnegative bacteria are shown in Table 1. From this it can be seen that of these compounds, ampicillin and benzylpenicillin alone showed appreciable activity. In general, ampicillin was two to ten times more active than benzylpenicillin, but certain strains were insensitive to both penicillins.

Table 1. The relative activities of seven penicillins against certain Gram-negative bacteria

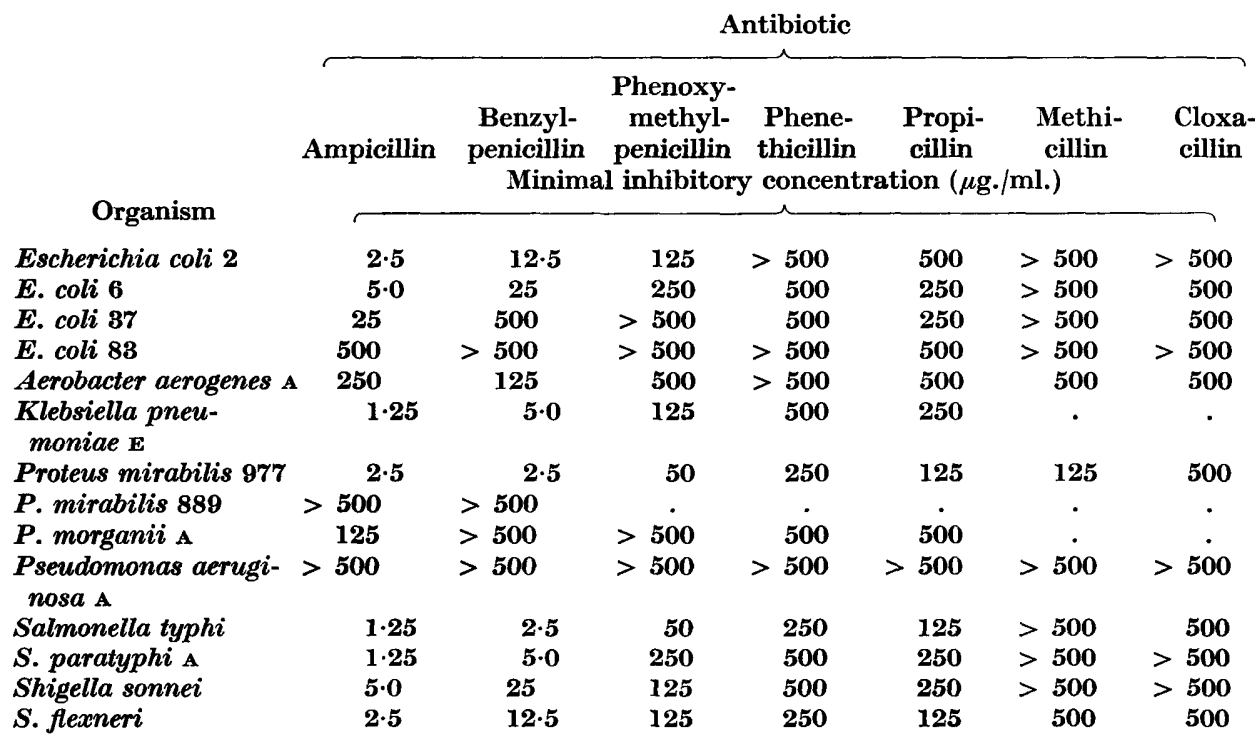

The relative stabilities of seven penicillins to the penicillinases of Gram-negative bacteria

The results of experiments designed to correlate the antibacterial activities of seven penicillins against two strains of Escherichia coli and the stabilities of these compounds to the penicillinases produced by the test bacteria are shown in Table 2 . In these tests the minimal inhibitory concentrations of the antibiotics were measured in the usual fashion in nutrient broth using an ampicillin-sensitive strain and an 


\section{Penicillin insensitivity}

ampicillin-resistant strain of $\boldsymbol{E}$. coli; in addition, the amount of destruction of penicillin which had occurred after overnight incubation was measured by microbiological assay. It will be seen that phenethicillin, propicillin, methicillin and cloxacillin were almost completely unaffected by the penicillinases produced by

Table 2. The relative stabilities of ampicillin, benzylpenicillin, phenoxymethylpenicillin, phenethicillin, propicillin, methicillin, and cloxacillin to the penicillinases produced by two strains of Escherichia coli

Minimal inhibitory concentrations of the penicillins against two strains of Escherichia coli were determined by serial dilution in nutrient broth; growth and residual penicillin activity after overnight incubation at $37^{\circ}$ were determined by visual inspection and microbiological assay respectively.

Penicillin Organism Growth and penicillin concentration $\left(\mu \mathrm{g} . / \mathrm{ml}\right.$.) after incubation at $37^{\circ}$ for $18 \mathrm{hr}$.

\begin{tabular}{|c|c|c|c|c|c|c|c|c|c|c|}
\hline \multicolumn{2}{|c|}{ 3acteria-free control* } & \multirow{2}{*}{$\begin{array}{r}500 \\
- \\
470\end{array}$} & \multirow{2}{*}{$\begin{array}{r}250 \\
- \\
245\end{array}$} & \multirow{2}{*}{$\begin{array}{r}125 \\
- \\
96\end{array}$} & \multirow{2}{*}{$\begin{array}{l}50 \\
- \\
45\end{array}$} & \multirow{2}{*}{$\begin{array}{l}25 \\
- \\
17 \cdot 2\end{array}$} & \multirow{2}{*}{$\begin{array}{l}12 \cdot 5 \\
+ \\
2 \cdot 2\end{array}$} & \multirow{2}{*}{$\begin{array}{c}5 \cdot 0 \\
+ \\
2 \cdot 2\end{array}$} & \multirow{2}{*}{$\begin{array}{c}2.5 \\
+ \\
0.9\end{array}$} & \multirow{2}{*}{$\begin{array}{l}1.25 \\
+ \\
0.4\end{array}$} \\
\hline $\begin{array}{l}\text { 3enzyl- } \\
\text { penicillin }\end{array}$ & E. coli 2 & & & & & & & & & \\
\hline & E. coli 83 & $\begin{array}{r}+ \\
0\end{array}$ & $\begin{array}{r}+ \\
0\end{array}$ & $\begin{array}{r}+ \\
0\end{array}$ & + & + & + & + & + & + \\
\hline \multirow[t]{2}{*}{ umpicillin } & E. coli 2 & $\overline{560}$ & $\overline{245}$ & $\overline{120}$ & $\overline{53}$ & $\overline{24 \cdot 0}$ & $\overline{13 \cdot 4}$ & $\overline{5 \cdot 3}$ & + & + \\
\hline & E. coli 83 & $\underset{400}{ \pm}$ & $\begin{array}{c}+ \\
82\end{array}$ & $\begin{array}{c}+ \\
24\end{array}$ & + & $\stackrel{+}{0 \cdot 7}$ & + & + & $\begin{array}{l}+ \\
0\end{array}$ & $\begin{array}{c}+ \\
0\end{array}$ \\
\hline \multirow{2}{*}{$\begin{array}{l}\text { 'henoxy- } \\
\text { methyl- } \\
\text { penicillin }\end{array}$} & E. coli 2 & $\underset{520}{ \pm}$ & $\stackrel{ \pm}{240}$ & $\underset{115}{+}$ & $\stackrel{+}{\mathbf{3 4}}$ & $\stackrel{+}{13 \cdot 0}$ & + & + & + & $\stackrel{+}{0 \cdot 6}$ \\
\hline & E. coli 83 & + & + & $\begin{array}{c}+ \\
0\end{array}$ & $\begin{array}{c}+ \\
0\end{array}$ & $\begin{array}{c}+ \\
0\end{array}$ & $\begin{array}{c}+ \\
0\end{array}$ & + & $\begin{array}{c}+ \\
0\end{array}$ & $\begin{array}{c}+ \\
0\end{array}$ \\
\hline \multirow[t]{2}{*}{$\begin{array}{l}\text { 'henethi- } \\
\text { cillin }\end{array}$} & E. coli 2 & $\begin{array}{r}+ \\
\mathbf{5 3 0}\end{array}$ & $\begin{array}{c}+ \\
\mathbf{2 5 0}\end{array}$ & $\begin{array}{r}+ \\
132\end{array}$ & $\underset{53}{+}$ & $\stackrel{+}{27 \cdot 0}$ & $\stackrel{+}{13 \cdot 0}$ & + & $+\underset{2 \cdot 6}{+}$ & + \\
\hline & E. coli 83 & $\stackrel{+}{550}$ & $\stackrel{+}{195}$ & $\begin{array}{c}+ \\
93\end{array}$ & $\begin{array}{c}+ \\
41\end{array}$ & $\stackrel{+}{16.5}$ & + & $+\underset{2 \cdot 6}{+}$ & $+\frac{+}{0 \cdot 6}$ & + \\
\hline \multirow[t]{2}{*}{ ropicillin } & E. coli 2 & $\stackrel{ \pm}{490}$ & $\begin{array}{r}+ \\
250\end{array}$ & $\begin{array}{r}+ \\
120\end{array}$ & $\stackrel{+}{45}$ & $\stackrel{+}{26 \cdot 0}$ & $\stackrel{+}{12 \cdot 0}$ & + & + & $\stackrel{+}{0.9}$ \\
\hline & E. coli 83 & $\underset{510}{ \pm}$ & $\begin{array}{r}+ \\
250\end{array}$ & $\stackrel{+}{127}$ & $\begin{array}{c}+ \\
40\end{array}$ & $\stackrel{+}{23 \cdot 0}$ & $\stackrel{+}{11 \cdot 0}$ & + & + & $\stackrel{+}{0.4}$ \\
\hline \multirow[t]{2}{*}{ lethicillin } & E. coli 2 & . & $\stackrel{+}{250}$ & $\stackrel{+}{128}$ & $\stackrel{+}{54}$ & $\stackrel{+}{25 \cdot 0}$ & $\stackrel{+}{13 \cdot 0}$ & + & $+\underset{2 \cdot 6}{+}$ & + \\
\hline & E. coli 83 & . & $\begin{array}{r}+ \\
250\end{array}$ & $\begin{array}{r}+ \\
130\end{array}$ & $\stackrel{+}{49}$ & $\stackrel{+}{25 \cdot 0}$ & $\begin{array}{l}+ \\
12.5\end{array}$ & + & + & $\stackrel{+}{0.9}$ \\
\hline \multirow[t]{2}{*}{ loxacillin } & E. coli 2 & . & $\stackrel{+}{235}$ & $\begin{array}{r}+ \\
128\end{array}$ & $\stackrel{+}{51}$ & $\stackrel{+}{24 \cdot 0}$ & $\stackrel{+}{13 \cdot 0}$ & + & + & + \\
\hline & E. coli 83 & . & $\begin{array}{r}+ \\
245\end{array}$ & $\begin{array}{r}+ \\
130\end{array}$ & $\begin{array}{c}+ \\
51\end{array}$ & $\stackrel{+}{25 \cdot 0}$ & $\begin{array}{l}+ \\
13 \cdot 0\end{array}$ & + & + & + \\
\hline
\end{tabular}

* These concentrations represent the initial concentrations of penicillins prior to incubation with the cultures.

$+=$ Growth.

- = No growth.

$\pm=$ Partial growth.

either strain of $\boldsymbol{E}$. coli, but exerted little or no inhibitory effect on these cultures. In contrast, there was complete destruction of benzylpenicillin and marked destruction of ampicillin by the resistant strain of $E$. coli strain 83, and there was some 
obvious destruction of benzylpenicillin by the sensitive strain of $E$. coli 2 at concentrations below the inhibitory value. Ampicillin was, however, unaffected by this strain. Phenoxymethylpenicillin appeared to occupy a mid-way position between these two groups of penicillins, inasmuch as this compound was almost inactive against one strain and was completely inactive against the other; but, nevertheless, there was obvious destruction of this penicillin by the ampicillinsensitive organism and complete destruction by the second strain.

Table 3. The activities of ampicillin and benzylpenicillin against Gram-negative. bacteria causing minimal destruction of penicillins

Minimal inhibitory concentrations of the penicillins against five strains of Gram-negative bacteria were determined by serial dilution in nutrient broth; growth and residual penicillin activity after overnight incubation at $37^{\circ}$ were determined by visual inspection and microbiological assay respectively.

Organism Antibiotic

Bacteria-free control*

$\begin{array}{cc}\text { Salmonella } & \text { Benzyl- } \\ \text { typhi } & \text { penicillin } \\ & \text { Ampicillin }\end{array}$

Growth and penicillin concentration $\left(\mu \mathrm{g} \cdot / \mathrm{ml}\right.$.) after incubation at $37^{\circ}$ for $18 \mathrm{hr}$.

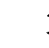

S. paratyphi A Benzylpenicillin

Ampicillin

\begin{tabular}{|c|c|c|c|c|c|c|c|}
\hline 50 & 25 & $12 \cdot 5$ & $5 \cdot 0$ & $2 \cdot 5$ & $1 \cdot 25$ & 0.5 & $0 \cdot 25$ \\
\hline
\end{tabular}

\begin{tabular}{|c|c|c|c|c|c|c|c|c|c|}
\hline & penicillin & & . & $5 \cdot 0$ & 1.9 & $1 \cdot 4$ & $0 \cdot 7$ & $0 \cdot 28$ & $0 \cdot 16$ \\
\hline & Ampicillin & & . & . & - & - & \pm & + & + \\
\hline $\begin{array}{l}\text { Proteus } \\
\text { mirabilis } 4\end{array}$ & $\begin{array}{l}\text { Benzyl- } \\
\text { penicillin }\end{array}$ & . & $\overline{12 \cdot 5}$ & $\overline{5 \cdot 0}$ & $\overline{2.0}$ & $\stackrel{+}{1.0}$ & $\begin{array}{l}+ \\
0.52\end{array}$ & $\stackrel{+}{0.29}$ & $\stackrel{+}{0 \cdot 16}$ \\
\hline & Ampicillin & . & - & 5 & $\overrightarrow{2 \cdot 6}$ & $\overline{1 \cdot 4}$ & $\overline{0.53}$ & $\stackrel{+}{+}$ & $\stackrel{+}{0 \cdot 16}$ \\
\hline $\begin{array}{l}\text { P. mirabilis } \\
\text { c977 }\end{array}$ & $\begin{array}{l}\text { Benzyl- } \\
\text { penicillin }\end{array}$ & . & - & $\overline{4 \cdot 2}$ & $\begin{array}{l}+ \\
1.9\end{array}$ & + & $\begin{array}{l}+ \\
0 \cdot 44\end{array}$ & $\stackrel{+}{+} \cdot$ & $\stackrel{+}{0 \cdot 16}$ \\
\hline & Ampicillin & . & $\dot{.}$ & $\overline{5.0}$ & $\overline{2 \cdot 6}$ & $\overline{1 \cdot 4}$ & $\stackrel{+}{0.53}$ & $\begin{array}{l}+ \\
0 \cdot 21\end{array}$ & $\stackrel{+}{0 \cdot 1}$ \\
\hline $\begin{array}{l}\text { Klebsiella } \\
\text { pneumoniae } \mathbf{E}\end{array}$ & $\begin{array}{l}\text { Benzyl- } \\
\text { penicillin }\end{array}$ & . & $\overline{10 \cdot 5}$ & $\begin{array}{l}- \\
5 \cdot 0\end{array}$ & $\overline{2 \cdot 4}$ & $\frac{ \pm}{1 \cdot 4}$ & $\begin{array}{l}+ \\
0 \cdot 45\end{array}$ & $\begin{array}{l}+ \\
0.28\end{array}$ & $<\stackrel{+}{0 \cdot 1}$ \\
\hline & Ampicillin & . & . & $\overline{5 \cdot 6}$ & - & $\overline{1 \cdot 3}$ & $\begin{array}{l}- \\
0 \cdot 6\end{array}$ & $\stackrel{+}{0 \cdot 23}$ & $\begin{array}{l}+ \\
<\cdot 1\end{array}$ \\
\hline
\end{tabular}

* These concentrations represent the initial concentrations of penicillins prior to incubation with the cultures.

$+=$ Growth.

$-=$ No growth.

$\pm=$ Partial growth.

The antibacterial activities and penicillinase stabilities of ampicillin and benzylpenicillin towards Gram-negative bacteria

As a result of numerous experiments to compare the relative antibacterial activities of ampicillin and benzylpenicillin, the Gram-negative bacteria were grouped as follows:

Group 1. Gram-negative bacteria sensitive to both ampicillin and benzylpenicillin. The results in Table 3 show the relative activities of ampicillin and benzylpenicillin against two Salmonella species, two strains of Proteus mirabilis and one strain of 
Klebsiella pneumoniae. These organisms were inhibited by concentrations of 0.5-5.0 $\mu$ g. penicillins/ml. but, in general, ampicillin was two to four times more active than was benzylpenicillin. Microbiological assay of these samples showed that there had been little or no destruction of either penicillin after incubation with the test organisms at $37^{\circ}$ for $16-20 \mathrm{hr}$.

Group 2. Gram-negative bacteria sensitive to ampicillin but relatively resistant to benzylpenicillin. From Table 4 it can be seen that ampicillin inhibited the growth of three strains of Escherichia coli and one strain of each of Shigella flexneri and

\section{Table 4. Penicillinase activities of certain Gram-negative bacteria sensitive to ampicillin but moderately insensitive to benzylpenicillin}

Minimal inhibitory concentrations of the penicillins against five strains of Gram-negative bacteria were determined by serial dilution in nutrient broth; growth and residual penicillin activity after overnight incubation at $37^{\circ}$ were determined by visual inspection and microbiological assay respectively.

Organism Antibiotic Growth and penicillin concentration ( $\mu$ g. $/ \mathrm{ml}$.) after incubation at $87^{\circ}$ for $18 \mathrm{hr}$.

\begin{tabular}{|c|c|c|c|c|c|c|c|c|c|c|}
\hline \multirow{2}{*}{\multicolumn{2}{|c|}{ 3acteria-free control* }} & \multirow{3}{*}{$\begin{array}{l}50 \\
. \\
.\end{array}$} & \multirow{3}{*}{$\begin{array}{c}25 \\
- \\
13 \cdot 5\end{array}$} & \multirow{3}{*}{$\begin{array}{r}12 \cdot 5 \\
\pm \\
13 \cdot 0\end{array}$} & \multirow{3}{*}{$\begin{array}{l}5 \cdot 0 \\
+ \\
0.44\end{array}$} & \multirow{3}{*}{$\begin{array}{l}\mathbf{2 . 5} \\
+ \\
0 \cdot 2\end{array}$} & \multirow{3}{*}{$\begin{array}{l}1 \cdot 25 \\
+ \\
0.16\end{array}$} & \multirow{3}{*}{$\begin{array}{l}0.5 \\
+ \\
0.12\end{array}$} & \multirow{3}{*}{$\begin{array}{c}0.25 \\
+ \\
<0.1\end{array}$} & \multirow{3}{*}{$\begin{array}{r}0.1 \\
+ \\
<0.1\end{array}$} \\
\hline & & & & & & & & & & \\
\hline $\begin{array}{l}\text { 'higella } \\
\text { flexneri }\end{array}$ & $\begin{array}{l}\text { Benzyl } \\
\text { penicillin }\end{array}$ & & & & & & & & & \\
\hline & Ampicillin & $\dot{.}$ & $\cdot$ & $\cdot$ & $\overline{5 \cdot 0}$ & $\overline{2 \cdot 6}$ & $\overline{1 \cdot 7}$ & $\begin{array}{l}+ \\
0 \cdot 44\end{array}$ & $\begin{array}{c}+ \\
\mathbf{0 \cdot 2}\end{array}$ & $\begin{array}{c}+ \\
<0 \cdot 1\end{array}$ \\
\hline \multirow[t]{2}{*}{ i. sonnei } & $\begin{array}{l}\text { Benzyl- } \\
\text { penicillin }\end{array}$ & - & \pm & \pm & $\stackrel{+}{0}$ & $\begin{array}{l}+ \\
\mathbf{0}\end{array}$ & $\stackrel{+}{0}$ & $\stackrel{+}{0}$ & $\stackrel{+}{0}$ & + \\
\hline & Ampicillin & $\dot{.}$ & $\dot{.}$ & $\dot{.}$ & $\frac{-}{5 \cdot 0}$ & $\overline{\mathbf{2} \cdot 1}$ & $\overline{1 \cdot 05}$ & $\stackrel{+}{0 \cdot 12}$ & $\begin{array}{c}+ \\
<\mathbf{0} \cdot 1\end{array}$ & $\stackrel{+}{0.1}$ \\
\hline \multirow[t]{2}{*}{$\begin{array}{l}\text { ischerichia } \\
\text { coli } 2\end{array}$} & $\begin{array}{c}\text { Benzyl- } \\
\text { penicillin }\end{array}$ & $\dot{.}$ & $\overline{17 \cdot 0}$ & $\stackrel{+}{1 \cdot 9}$ & $\stackrel{+}{0 \cdot 64}$ & $\begin{array}{l}+ \\
0.46\end{array}$ & $\underset{1 \cdot 8}{+}$ & $\stackrel{+}{0.12}$ & $\begin{array}{c}+ \\
<0.1\end{array}$ & $\begin{array}{c}+ \\
0.1\end{array}$ \\
\hline & Ampicillin & . & $\dot{.}$ & . & 7 & $\overline{2 \cdot 6}$ & $\frac{ \pm}{1 \cdot 4}$ & $\stackrel{+}{0.53}$ & $\stackrel{+}{0 \cdot 14}$ & $\begin{array}{c}+ \\
<0 \cdot 1\end{array}$ \\
\hline \multirow[t]{2}{*}{ i. $\operatorname{coli} 61$} & $\begin{array}{l}\text { Benzyl- } \\
\text { penicillin }\end{array}$ & . & $\frac{-}{20 \cdot 0}$ & $\overline{6 \cdot 8}$ & $\stackrel{+}{\mathbf{0} \cdot 34}$ & $\mathbf{0}^{+}$ & $\mathbf{0}^{+}$ & $\stackrel{+}{0.12}$ & $\stackrel{+}{0}$ & $\begin{array}{l}+ \\
0\end{array}$ \\
\hline & Ampicillin & $\dot{.}$ & $\cdot$ & $\cdot$ & $\frac{-}{5 \cdot 0}$ & $\overline{2 \cdot 6}$ & $\overline{1 \cdot 4}$ & $\stackrel{+}{0 \cdot 41}$ & $\stackrel{+}{0.22}$ & $\begin{array}{c}+ \\
<0 \cdot 1\end{array}$ \\
\hline \multirow[t]{2}{*}{ i. coli $\mathbf{T} 341$} & $\begin{array}{l}\text { Benzyl- } \\
\text { penicillin }\end{array}$ & - & $\overline{27 \cdot 0}$ & $\underset{1 \cdot 5}{+}$ & $\underset{\mathbf{0}}{+}$ & $\underset{\mathbf{0}}{+}$ & $\begin{array}{l}+ \\
\mathbf{0}\end{array}$ & $\underset{0}{+}$ & $\underset{\mathbf{0}}{+}$ & $\underset{\mathbf{0}}{+}$ \\
\hline & Ampicillin & $\dot{.}$ & . & $\overline{15 \cdot 0}$ & $\overline{4 \cdot 4}$ & $\underset{\mathbf{2 \cdot 6 5}}{ \pm}$ & $\stackrel{+}{0 \cdot 63}$ & $\begin{array}{l}+ \\
0 \cdot 24\end{array}$ & $\mathbf{0}^{+}$ & + \\
\hline
\end{tabular}

* These concentrations represent the initial concentrations of penicillins prior to incubation with the cultures.

$+=$ Growth.

$-=$ No growth.

$\pm=$ Partial growth.

S. sonnei at concentrations of $1 \cdot 25-2 \cdot 5 \mu \mathrm{g} . / \mathrm{ml}$, whereas benzylpenicillin was inhibitory only at 12.5-25 $\mu \mathrm{g}$. $/ \mathrm{ml}$., thus showing a tenfold difference in the activities of these penicillins. Moreover, all five strains of bacteria destroyed most or all of the benzylpenicillin at concentrations below the minimal inhibitory concentrations, but there was little or no destruction of ampicillin by these organisms. These results showed the greater stability of ampicillin to the penicillinases produced by these bacteria, and suggested the possibility that the sensitivity of each strain to these 
penicillins was related to the stabilities of the compounds to the penicillinase elaborated by each particular strain. This is supported by the results of an experiment shown in Fig. 1, illustrating the growth of one of these strains of bacteria, $E$. coli $\mathbf{T 3 4 1 ,}$ in the presence of different concentrations of benzylpenicillin, and in Table 5 which shows the rate of destruction of the penicillin by this strain. It can be seen that at the lowest benzylpenicillin concentration $(5 \mu \mathrm{g} . / \mathrm{ml}$.) growth was as rapid as in the control tube containing no antibiotic, while the concentration of antibiotic diminished steadily until none could be detected after $7 \mathrm{hr}$. At the next higher concentration $(12.5 \mu \mathrm{g} . / \mathrm{ml}$.), there was little increase in growth for the first

\section{Table 5. Destruction of benzylpenicillin by cultures of Escherichia coli $r 341$}

Nutrient broth solutions containing graded concentrations of benzylpenicillin were inoculated with Escherichia coli $\mathrm{T341}$ and incubated at $37^{\circ}$ for $24 \mathrm{hr}$.; samples were taken at intervals and assayed for penicillin content by microbiological assay.

\begin{tabular}{crcc}
$\begin{array}{c}\text { Incubation } \begin{array}{c}\text { time } \\
\text { (hr) }\end{array} \\
0\end{array}$ & \multicolumn{3}{c}{$\begin{array}{c}\text { Penicillin concentration } \\
(\mu \mathrm{gg} \cdot / \mathrm{ml} .)\end{array}$} \\
\cline { 2 - 4 } 1 & 25 & $12 \cdot 5$ & $5 \cdot 0$ \\
2 & 24 & $11 \cdot 5$ & $5 \cdot 4$ \\
3 & 20 & $9 \cdot 2$ & $5 \cdot 0$ \\
4 & 16 & $6 \cdot 3$ & $4 \cdot 5$ \\
5 & 15 & $3 \cdot 0$ & $2 \cdot 5$ \\
6 & 12 & $1 \cdot 3$ & $1 \cdot 3$ \\
7 & 11 & $1 \cdot 3$ & $0 \cdot 9$ \\
24 & 10 & 0 & 0 \\
& 0 & 0 & 0
\end{tabular}

3-4 $\mathrm{hr}$., while the penicillin concentration fell gradually to $3.0 \mu \mathrm{g} . / \mathrm{ml}$.; during the next $3 \mathrm{hr}$ the turbidity of the culture increased steadily and the penicillin concentration fell to zero. At the highest concentration $(25 \mu \mathrm{g} . / \mathrm{ml}$. $)$, there was no increase in turbidity over a period of $7 \frac{1}{2} \mathrm{hr}$, during which time the penicillin concentration decreased from 25 to $10 \mu \mathrm{g}$. $/ \mathrm{ml}$. After incubation overnight at $37^{\circ}$ the turbidities of all these cultures were approximately equivalent to that of the control culture containing no penicillin, and no penicillin was detected for any concentration.

Group 3. Gram-negative bacteria resistant to both ampicillin and benzylpenicillin. Examples of bacteria resistant to ampicillin and to benzylpenicillin are shown in Table 6; it can be seen that all strains brought about marked destruction of both penicillins. In some cases, no penicillin could be detected in any of the culture tubes in which there was growth, e.g. Escherichia coli 70, Proteus mirabilis 889, Klebsiella pneumoniae c, Aerobacter aerogenes $\mathrm{A}$ and G. In other cases there was complete destruction of benzylpenicillin, but only partial destruction of ampicillin, for example, with strains of $\boldsymbol{P}$. rettgeri $\mathrm{A}$ and $\boldsymbol{P}$ s. aeruginosa $\mathrm{A}$ and $\mathrm{C}$. Yet again, with some strains there was only partial destruction of either penicillin, e.g. E. coli 37 and Paracolon wM 29.

Quantitative estimation of penicillinase activity by Pseudomonas aeruginosa was made by bioautographic techniques, as it was noted that antibiotic-free cultures of this species displayed marked antibacterial activity against the assay organism, Bacillus subtilis ATcc 6633, with the result that microbiological assays showed little 
Table 6. Penicillinase activities of Gram-negative bacteria resistant to ampicillin and benzylpenicillin

Minimal inhibitory concentrations of the penicillins against ten strains of Gram-negative bacteria were determined by serial dilution in nutrient broth; growth and residual penicillin activity after overnight incubation at $37^{\circ}$ were determined by visual inspection and microbiological assay respectively.

Organism Antibiotic Growth and penicillin concentration ( $\mu \mathrm{g} . / \mathrm{ml}$.) after incubation at $37^{\circ}$ for $18 \mathrm{hr}$.

\begin{tabular}{|c|c|c|c|c|c|c|c|c|c|c|}
\hline \multirow{2}{*}{\multicolumn{2}{|c|}{ Bacteria-free control* }} & \multirow{3}{*}{$\begin{array}{c}500 \\
. \\
.\end{array}$} & \multirow{3}{*}{$\begin{array}{r}250 \\
+ \\
0\end{array}$} & \multirow{3}{*}{$\begin{array}{r}125 \\
+ \\
0\end{array}$} & \multirow{3}{*}{$\begin{array}{r}50 \\
+ \\
0\end{array}$} & \multirow{3}{*}{$\begin{array}{r}25 \\
+ \\
0\end{array}$} & & & & \\
\hline & & & & & & & \multirow{2}{*}{$\begin{array}{l}12 \cdot 5 \\
+ \\
0\end{array}$} & \multirow{2}{*}{$\begin{array}{l}5 \cdot 0 \\
+ \\
0\end{array}$} & \multirow{2}{*}{$\begin{array}{l}\mathbf{2 . 5} \\
+ \\
0\end{array}$} & \multirow{2}{*}{$\begin{array}{l}\mathbf{1} \cdot \mathbf{2 5} \\
+ \\
0\end{array}$} \\
\hline $\begin{array}{l}\text { Escherichia } \\
\text { coli } 70\end{array}$ & $\begin{array}{l}\text { Benzyl- } \\
\text { penicillin }\end{array}$ & & & & & & & & & \\
\hline & Ampicillin & $\dot{ }$ & + & $\begin{array}{c}+ \\
0\end{array}$ & $\begin{array}{l}+ \\
0\end{array}$ & $\begin{array}{c}+ \\
0\end{array}$ & $\begin{array}{c}+ \\
0\end{array}$ & $\begin{array}{c}+ \\
0\end{array}$ & $\begin{array}{l}+ \\
0\end{array}$ & $\begin{array}{r}+ \\
0\end{array}$ \\
\hline \multirow{2}{*}{$\begin{array}{l}\text { Proteus } \\
\text { mirabilis } \\
\mathbf{8 8 9}\end{array}$} & $\begin{array}{l}\text { Benzyl- } \\
\text { penicillin }\end{array}$ & $\dot{.}$ & $\begin{array}{r}+ \\
0\end{array}$ & $\begin{array}{r}+ \\
0\end{array}$ & $\begin{array}{c}+ \\
0\end{array}$ & $\begin{array}{r}+ \\
0\end{array}$ & $\begin{array}{r}+ \\
\mathbf{0}\end{array}$ & $\begin{array}{r}+ \\
0\end{array}$ & $\begin{array}{c}+ \\
0\end{array}$ & $\begin{array}{r}+ \\
\mathbf{0}\end{array}$ \\
\hline & Ampicillin & $\dot{.}$ & $\begin{array}{r}+ \\
0\end{array}$ & $\begin{array}{c}+ \\
0\end{array}$ & $\begin{array}{c}+ \\
0\end{array}$ & $\begin{array}{r}+ \\
0\end{array}$ & + & $\begin{array}{r}+ \\
0\end{array}$ & $\begin{array}{c}+ \\
0\end{array}$ & $\begin{array}{r}+ \\
0\end{array}$ \\
\hline \multirow{2}{*}{$\begin{array}{l}\text { Klebsiella } \\
\text { pneumoniae } \\
\text { C }\end{array}$} & $\begin{array}{l}\text { Benzyl- } \\
\text { penicillin }\end{array}$ & . & $\begin{array}{c}+ \\
0\end{array}$ & $\begin{array}{c}+ \\
0\end{array}$ & + & $\begin{array}{r}+ \\
0\end{array}$ & $\begin{array}{r}+ \\
0\end{array}$ & + & $\begin{array}{r}+ \\
0\end{array}$ & + \\
\hline & Ampicillin & $\dot{.}$ & $\begin{array}{c}+ \\
0\end{array}$ & $\begin{array}{r}+ \\
0\end{array}$ & $\begin{array}{r}+ \\
0\end{array}$ & $\begin{array}{c}+ \\
0\end{array}$ & $\begin{array}{r}+ \\
0\end{array}$ & $\begin{array}{r}+ \\
0\end{array}$ & + & + \\
\hline \multirow[t]{2}{*}{$\begin{array}{c}\text { Aerobacter } \\
\text { aerogenes A }\end{array}$} & $\begin{array}{l}\text { Benzyl- } \\
\text { penicillin }\end{array}$ & . & $\overline{170}$ & $\overline{8}$ & $\begin{array}{r}+ \\
0\end{array}$ & $\begin{array}{r}+ \\
0\end{array}$ & $\begin{array}{r}+ \\
0\end{array}$ & $\begin{array}{r}+ \\
0\end{array}$ & $\begin{array}{c}+ \\
0\end{array}$ & $\begin{array}{r}+ \\
0\end{array}$ \\
\hline & Ampicillin & . & $\overline{90}$ & $\begin{array}{c}+ \\
0\end{array}$ & $\begin{array}{r}+ \\
0\end{array}$ & $\begin{array}{c}+ \\
0\end{array}$ & $\begin{array}{r}+ \\
0\end{array}$ & $\begin{array}{c}+ \\
0\end{array}$ & + & $\begin{array}{r}+ \\
0\end{array}$ \\
\hline \multirow[t]{2}{*}{$\begin{array}{l}\text { A. aerogenes } \\
\mathbf{G}\end{array}$} & $\begin{array}{l}\text { Benzyl- } \\
\text { penicillin }\end{array}$ & $\dot{ }$ & $\overline{55}$ & $\begin{array}{r}+ \\
0\end{array}$ & $\begin{array}{c}+ \\
0\end{array}$ & $\begin{array}{r}+ \\
0\end{array}$ & $\begin{array}{r}+ \\
0\end{array}$ & $\begin{array}{r}+ \\
0\end{array}$ & $\begin{array}{r}+ \\
0\end{array}$ & $\begin{array}{r}+ \\
0\end{array}$ \\
\hline & Ampicillin & . & $\begin{array}{c}+ \\
0\end{array}$ & $\begin{array}{c}+ \\
0\end{array}$ & $\begin{array}{c}+ \\
0\end{array}$ & $\begin{array}{r}+ \\
0\end{array}$ & $\begin{array}{r}+ \\
0\end{array}$ & $\begin{array}{c}+ \\
0\end{array}$ & $\begin{array}{r}+ \\
0\end{array}$ & + \\
\hline \multirow{2}{*}{$\begin{array}{l}\text { Pseudomonas } \\
\text { aeruginosa } \\
\text { A }\end{array}$} & $\begin{array}{l}\text { Benzyl- } \\
\text { penicillin }\end{array}$ & $\begin{array}{c}+ \\
0\end{array}$ & $\begin{array}{c}+ \\
0\end{array}$ & + & $\begin{array}{l}+ \\
.\end{array}$ & + & $\begin{array}{l}+ \\
.\end{array}$ & + & + & $\begin{array}{l}+ \\
.\end{array}$ \\
\hline & Ampicillin & $\begin{array}{r}+ \\
530\end{array}$ & $\begin{array}{c}+ \\
135\end{array}$ & $\begin{array}{l}+ \\
.\end{array}$ & $\begin{array}{l}+ \\
.\end{array}$ & $\begin{array}{l}+ \\
.\end{array}$ & $\begin{array}{l}+ \\
.\end{array}$ & + & $\begin{array}{l}+ \\
.\end{array}$ & $\begin{array}{l}+ \\
.\end{array}$ \\
\hline \multirow[t]{2}{*}{$\begin{array}{l}\text { Ps. aeru- } \\
\text { ginosa c9 }\end{array}$} & $\begin{array}{l}\text { Benzyl- } \\
\text { penicillin }\end{array}$ & $\begin{array}{c}+ \\
0\end{array}$ & $\begin{array}{c}+ \\
0\end{array}$ & $\begin{array}{l}+ \\
.\end{array}$ & $\begin{array}{l}+ \\
.\end{array}$ & + & $\begin{array}{l}+ \\
\text {. }\end{array}$ & $\begin{array}{l}+ \\
.\end{array}$ & + & + \\
\hline & Ampicillin & $\begin{array}{r}+ \\
375\end{array}$ & $\begin{array}{r}+ \\
150\end{array}$ & $\begin{array}{l}+ \\
.\end{array}$ & $\begin{array}{l}+ \\
.\end{array}$ & $\begin{array}{l}+ \\
.\end{array}$ & $\begin{array}{l}+ \\
\text {. }\end{array}$ & $\begin{array}{l}+ \\
.\end{array}$ & $\begin{array}{l}+ \\
.\end{array}$ & $\begin{array}{l}+ \\
.\end{array}$ \\
\hline \multirow[t]{2}{*}{$\begin{array}{l}\text { Proteus } \\
\text { rettgeri A }\end{array}$} & $\begin{array}{l}\text { Benzyl- } \\
\text { penicillin }\end{array}$ & $\begin{array}{c}+ \\
0\end{array}$ & $\begin{array}{c}+ \\
0\end{array}$ & + & $\begin{array}{c}+ \\
\mathbf{0}\end{array}$ & $\begin{array}{c}+ \\
0\end{array}$ & + & + & + & $\begin{array}{r}+ \\
0\end{array}$ \\
\hline & Ampicillin & $5 \overline{20}$ & 260 & $\overline{120}$ & $\frac{ \pm}{40}$ & $\stackrel{+}{9 \cdot 2}$ & $\stackrel{+}{0.88}$ & + & + & $\begin{array}{r}+ \\
0\end{array}$ \\
\hline \multirow[t]{2}{*}{ E. coli 37} & $\begin{array}{l}\text { Benzyl- } \\
\text { penicillin }\end{array}$ & $+{ }_{8 \cdot 5}^{+}$ & $\stackrel{+}{18 \cdot 5}$ & $\stackrel{+}{12.5}$ & + & $\stackrel{+}{0 \cdot 3}$ & $\stackrel{+}{<0.1}$ & $\stackrel{+}{0.5}$ & $\stackrel{+}{0 \cdot 4}$ & $\stackrel{+}{<0.1}$ \\
\hline & Ampicillin & 505 & $2 \overline{20}$ & $\begin{array}{r}+ \\
105\end{array}$ & $\begin{array}{c}+ \\
\mathbf{3 1}\end{array}$ & $\stackrel{+}{13 \cdot 0}$ & + & + & + & + \\
\hline \multirow[t]{2}{*}{$\begin{array}{l}\text { Paracolon } \\
\text { WM } 29\end{array}$} & $\begin{array}{l}\text { Benzyl- } \\
\text { penicillin }\end{array}$ & $\begin{array}{r}+ \\
160\end{array}$ & $\underset{72}{+}$ & $\underset{15}{+}$ & $\begin{array}{c}+ \\
15\end{array}$ & + & $\begin{array}{r}+ \\
0\end{array}$ & + & + & + \\
\hline & Ampicillin & $\begin{array}{r}+ \\
330\end{array}$ & $\stackrel{+}{135}$ & $\begin{array}{c}+ \\
82\end{array}$ & $\begin{array}{c}+ \\
29\end{array}$ & $\stackrel{+}{9 \cdot 3}$ & + & $+_{1.5}^{+}$ & $\stackrel{+}{0.35}$ & $\begin{array}{l}+ \\
0.1\end{array}$ \\
\hline
\end{tabular}

* These concentrations represent the initial concentrations of penicillins prior to incubation with the cultures. $+=$ Growth. - = No growth. $\pm=$ Partial growth. 
or no destruction of either penicillin by this species. In fact, most strains of $\boldsymbol{P}$. aeruginosa were capable of causing some destruction of both penicillins, ampicillin being more resistant than benzylpenicillin.

It is of interest that of some 30 strains of Gram-negative bacteria tested which were resistant to ampicillin and benzylpenicillin, all were capable of causing some degree of destruction of penicillin. In this series, no organisms which did not produce penicillinase and were inherently penicillin insensitive were isolated. In general, ampicillin was more stable than benzylpenicillin to the penicillinases produced by these Gram-negative bacteria, save that strains of Aerobacter aerogenes appeared to destroy ampicillin to a rather greater extent than benzylpenicillin.

Further experiments were designed to attempt to determine to what extent the resistance of these bacteria was due to penicillinase production. A simple type of experiment appeared to be estimation of the effect of decrease in inoculum size on

Table 7. The effect of alteration of inoculum size on the sensitivity of Gramnegative bacteria to ampicillin and benzylpenicillin

\begin{tabular}{|c|c|c|c|}
\hline \multirow{3}{*}{$\begin{array}{c}\text { Organism } \\
\text { Escherichia coli } 70\end{array}$} & \multirow[b]{2}{*}{ Inoculum* } & \multirow{2}{*}{\multicolumn{2}{|c|}{$\begin{array}{c}\text { Ampicillin penicillin } \\
\text { Minimal inhibitory } \\
\text { concentrations }(\mu \mathrm{g} . / \mathrm{ml} .)\end{array}$}} \\
\hline & & & \\
\hline & $\begin{array}{l}\text { Neat } \\
10^{-3} \\
10^{-6}\end{array}$ & $\begin{array}{r}>500 \\
12 \cdot 5 \\
12 \cdot 5\end{array}$ & $\begin{array}{r}500 \\
25 \\
25\end{array}$ \\
\hline Proteus vulgaris D & $\begin{array}{l}\text { Neat } \\
10^{-3} \\
10^{-6}\end{array}$ & $\begin{aligned}>500 \\
\quad 5 \cdot 0 \\
1 \cdot 25\end{aligned}$ & $\begin{array}{r}>\mathbf{5 0 0} \\
12 \cdot 5 \\
2 \cdot 5\end{array}$ \\
\hline P. morganii A & $\begin{array}{l}\text { Neat } \\
10^{-3} \\
10^{-6}\end{array}$ & $\begin{array}{c}125 \\
25 \\
2 \cdot 5\end{array}$ & $\begin{array}{l}>500 \\
125 \\
12 \cdot 5\end{array}$ \\
\hline Aerobacter aerogenes A & $\begin{array}{l}\text { Neat } \\
10^{-3} \\
10^{-6}\end{array}$ & $\begin{array}{r}500 \\
12 \cdot 5 \\
2 \cdot 5\end{array}$ & $\begin{array}{r}125 \\
12 \cdot 5 \\
5 \cdot 0\end{array}$ \\
\hline A. aerogenes $\mathrm{G}$ & $\begin{array}{l}\text { Neat } \\
10^{-3} \\
10^{-6}\end{array}$ & $\begin{array}{r}500 \\
12 \cdot 5 \\
2 \cdot 5\end{array}$ & $\begin{array}{r}125 \\
12 \cdot 5 \\
5 \cdot 0\end{array}$ \\
\hline Pseudomonas aeruginosa $\mathrm{A}$ & $\begin{array}{l}\text { Neat } \\
10^{-3} \\
10^{-6}\end{array}$ & $\begin{array}{r}\mathbf{5 0 0} \\
500 \\
500\end{array}$ & $\begin{array}{l}>500 \\
>500 \\
>500\end{array}$ \\
\hline E. coli 83 & $\begin{array}{l}\text { Neat } \\
10^{-8} \\
10^{-6}\end{array}$ & $\begin{array}{r}>500 \\
>500 \\
500\end{array}$ & $\begin{array}{l}>\mathbf{5 0 0} \\
>\mathbf{5 0 0} \\
>\mathbf{5 0 0}\end{array}$ \\
\hline P. mirabilis 889 & $\begin{array}{l}\text { Neat } \\
10^{-3} \\
10^{-6}\end{array}$ & $\begin{array}{l}500 \\
500 \\
250\end{array}$ & $\begin{array}{l}500 \\
250 \\
250\end{array}$ \\
\hline
\end{tabular}

the sensitivity of the organism to penicillin, the rationale being that intrinsically sensitive, penicillinase-producing bacteria would show a marked increase in sensitivity to penicillins with smaller inocula, whereas very small inocula of inherently insensitive organisms would still be capable of proliferating in high 
concentrations of penicillin. It can be seen from Table 7 that Escherichia coli 70, Proteus vulgaris $\mathrm{D}$ and $\boldsymbol{P}$. morganii A, and Aerobacter aerogenes $\mathrm{A}$ and $\mathrm{G}$ were very much more sensitive to ampicillin and benzylpenicillin when small inocula were employed, whereas with $P$ seudomonas aeruginosa $\mathrm{A}, \boldsymbol{E}$. coli 83, and $\boldsymbol{P}$. mirabilis 889 there was little or no change in sensitivity to ampicillin or benzylpenicillin, even when the inoculum was diminished by a factor of one in a million. From these results it would seem that the former five strains might be examples of intrinsically sensitive, penicillinase-producing bacteria, and that the latter three strains might serve as examples of intrinsically insensitive organisms. These results prompted further experiments designed to confirm these findings by correlating growth with concomitant penicillin destruction.

\section{Table 8. Destruction of benzylpenicillin by cultures of Aerobacter aerogenes $A$}

\begin{tabular}{|c|c|c|c|}
\hline \multirow{2}{*}{$\begin{array}{c}\text { Incubation } \\
\text { time } \\
(\mathrm{hr}) \\
0\end{array}$} & \multicolumn{3}{|c|}{$\begin{array}{c}\text { Penicillin concentration } \\
(\mu \mathrm{g} . / \mathrm{ml} .)\end{array}$} \\
\hline & 100 & 25 & $5 \cdot 0$ \\
\hline 1 & 100 & 25 & $4 \cdot 3$ \\
\hline 21 & 90 & 20 & $2 \cdot 6$ \\
\hline 4 & $\mathbf{3 7}$ & $2 \cdot 8$ & $1 \cdot 6$ \\
\hline $5 \frac{1}{2}$ & 4.7 & $2 \cdot 2$ & 0.7 \\
\hline 24 & 0 & 0 & 0 \\
\hline
\end{tabular}

An example of an intrinsically sensitive, but penicillinase-producing organism is illustrated in Fig. 2, which shows the growth of Aerobacter aerogenes A in the presence of different concentrations of benzylpenicillin; the rate of destruction of the penicillin by this culture is shown in Table 8. The minimal inhibitory concentration of this culture after overnight incubation was $500 \mu \mathrm{g}$. benzylpenicillin/ml., but during the first $6 \mathrm{hr}$ a concentration of $100 \mu \mathrm{g} . / \mathrm{ml}$. completely inhibited growth; during this period the penicillin concentration fell from 100 to $5 \mu \mathrm{g} . / \mathrm{ml}$. Thereafter growth took place so that after $24 \mathrm{hr}$ the turbidity of this culture equalled that of a penicillin-free control culture. These results appear to confirm that the growth of this culture in the presence of benzylpenicillin was related to the rate of destruction of the antibiotic by the organism.

In contrast, an intrinsically insensitive strain of Escherichia coli 83 multiplied in the presence of concentrations of up to $500 \mu \mathrm{g}$. benzylpenicillin $/ \mathrm{ml}$. as rapidly as did an antibiotic-free culture (Fig. 3), although the rate of destruction of penicillin was relatively slow (Table 9), showing that the organism was able to proliferate readily in the presence of high concentrations of penicillin.

Thus, the inferences drawn from the inoculum-dilution experiments regarding the nature of the insensitivity of various Gram-negative bacteria to penicillins appeared to be supported by the growth experiments illustrated above, and would seem to confirm that whereas some penicillinase-producing strains of Gramnegative bacteria are inherently insensitive to penicillins, with other strains resistance is due mainly to destruction of the antibiotic. 


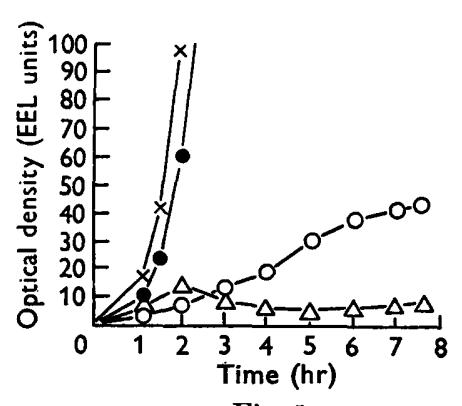

Fig. 1

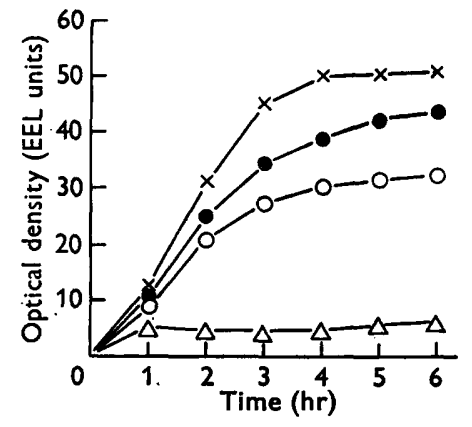

Fig. 2

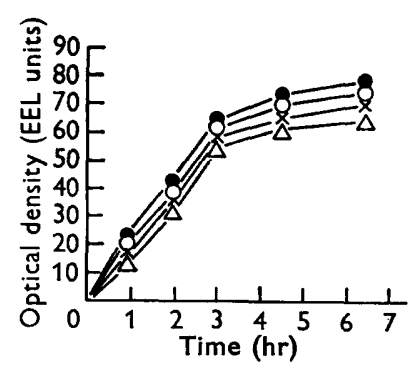

Fig. 3

Fig. 1. Growth of Escherichia coli (T 341) in the presence of benzylpenicillin. $\times$, Penicillin-free culture; $O$, culture containing penicillin, $5 \mu \mathrm{g} . / \mathrm{ml}$.; $O$, culture containing penicillin, 12.5 $\mu \mathrm{g} . / \mathrm{ml}$; $\Delta$, culture containing penicillin, $25 \mu \mathrm{g} . / \mathrm{ml}$.

Fig. 2. Growth of Aerobacter aerogenes A in the presence of benzylpenicillin. $\times$, Penicillinfree culture; $O$, culture containing penicillin, $5 \mu \mathrm{g} . / \mathrm{ml}$; $O$, culture containing penicillin, $25 \mu \mathrm{g} . / \mathrm{ml}$.; $\triangle$, culture containing penicillin, $100 \mu \mathrm{g} . / \mathrm{ml}$.

Fig. 3. Growth of Escherichia coli (83) in the presence of benzylpenicillin. $\times$, Penicillinfree culture; $O$, culture containing penicillin, $25 \mu \mathrm{g} . / \mathrm{ml}$.; $O$, culture containing penicillin, $125 \mu \mathrm{g} . / \mathrm{ml}$; $\Delta$, culture containing penicillin, $500 \mu \mathrm{g} . / \mathrm{ml}$.

Table 9. Destruction of benzylpenicillin by cultures of Escherichia coli 83

Nutrient broth solutions containing graded concentrations of benzylpenicillin were inoculated with Escherichia coli 83 and incubated at $37^{\circ}$ for $24 \mathrm{hr}$; samples were taken at intervals and assayed for penicillin content by microbiological assay.

Incubation
time
(hr)
0
1
$\mathbf{3}_{\frac{1}{2}}$
$\mathbf{5}_{\frac{1}{2}}$
$\mathbf{2 4}$

\begin{tabular}{|c|c|c|}
\hline \multicolumn{3}{|c|}{$\begin{array}{l}\text { Penicillin concentration } \\
(\mu \mathrm{g} . / \mathrm{ml} .)\end{array}$} \\
\hline 500 & 125 & 25 \\
\hline 490 & 124 & 20 \\
\hline 465 & 115 & 16 \\
\hline 425 & 90 & 8 \\
\hline 0 & o & 0 \\
\hline
\end{tabular}

\section{DISCUSSION}

On the basis of the results reported here, the penicillins which display a relatively low order of activity against any of the Gram-negative bacteria examined may be readily distinguished from those penicillins which show a significant effect against certain strains. Compounds in the former group include phenethicillin, propicillin, methicillin and cloxacillin, and it is plain that the insensitivity of the Gramnegative bacteria to these penicillins is in no way associated with penicillinase. In contrast, the sensitivities of Gram-negative bacteria to ampicillin and benzylpenicillin were very varied, and in many cases the reaction of the organism to these drugs appeared to depend upon the ability of the organism to produce a penicillinase capable of destroying the specific penicillin. From the experiments described above, the bacteria tested could be divided into three groups, according to the effect of the specific penicillinase upon ampicillin or benzylpenicillin, e.g.:

(a) Strains which produced little or no penicillinase and were sensitive to 
ampicillin and to benzylpenicillin, e.g. one Salmonella species, and some strains of Proteus mirabilis and Klebsiella.

(b) Strains which caused little or no destruction of ampicillin but more marked destruction of benzylpenicillin, and which were, accordingly, sensitive to ampicillin but less sensitive to benzylpenicillin, e.g. strains of Escherichia coli, Shigella species.

(c) Strains which were capable of destroying both compounds. This group, comprising penicillin-resistant organisms, could be further divided into: (i) intrinsically sensitive, penicillinase-producing, bacteria, e.g. strains of Aerobacter aerogenes, $\boldsymbol{P}$. vulgaris, $\boldsymbol{P}$. morganii; (ii) intrinsically insensitive, penicillinase-producing, bacteria, e.g. Pseudomonas aeruginosa, strains of $\boldsymbol{E}$. coli and $\boldsymbol{P}$. mirabilis.

It follows from these findings that the main difference in the activities of ampicillin and benzylpenicillin is associated with the increased stability of ampicillin towards the penicillinases produced by certain Gram-negative bacteria. Consequently, while ampicillin is from two to four times more active than benzylpenicillin against Gram-negative bacteria which produce little or no penicillinase, it is at least ten times more active against many strains which do produce penicillinase. The latter would apparently include the large number of strains of Escherichia coli which are sensitive to relatively low concentrations of ampicillin, but which require considerably higher concentrations of benzylpenicillin for inhibition. It is worth noting, however, that with these organisms growth is dependent upon destruction of the penicillin in the culture medium, so that benzylpenicillin might seem to be considerably more effective if antibacterial activity were measured over a shorter period of time than is conventional. Strains resistant to both penicillins are usually capable of destroying both compounds, although, again, ampicillin is generally more stable to the action of the penicillinases produced by these organisms. An exception to this finding is that benzylpenicillin appears to be more stable to Aerobacter aerogenes than is ampicillin but there is usually extensive destruction of both compounds. These findings are in good agreement with those of various other workers who have reported that ampicillin displays increased stability to the penicillinases produced by Gram-negative bacteria (Auhagen et al. 1962; Ayliffe, 1963; Smith, 1963). The last two workers also noted the superior stability of benzylpenicillin to the penicillinases produced by Aerobacter species.

In this series of experiments, all the resistant strains of bacteria produced penicillinase, whereas Trafford et al. (1962) reported that a relatively high proportion of bacteria which did not produce penicillinase were resistant to ampicillin. On the other hand, Barber (1962) reported that the majority of coliform bacteria are penicillinase-producing organisms capable of destroying penicillins. It is possible that in some cases at least, divergences in findings may be due to differences in techniques; this is being investigated. Likewise, although Knox \& Smith (1962) have described strains of Gram-negative bacteria which are sensitive to ampicillin but with inherent resistance to benzylpenicillin, such strains have not been isolated in the experiments described above.

It thus seems that benzylpenicillin is active against strains of Gram-negative bacteria which produce little or no penicillinase, but is ineffective against penicillinase-producing bacteria. In contrast, ampicillin is relatively stable to the penicillinases produced by certain of the Gram-negative bacteria, and is, as a consequence, substantially more active than benzylpenicillin against many of these 
bacteria. With resistant strains of bacteria, however, the situation is much more complex; here insensitivity may be due to penicillinase activity alone, or may be due to inherent resistance, although in the results reported here this inherent resistance was always accompanied by some degree of penicillin destruction.

It is a pleasure to thank $\operatorname{Dr}$ M. O. Moss for his assistance in carrying out the chromatographic procedures and Dr G. N. Rolinson for much helpful advice and criticism.

\section{REFERENCES}

Abraham, E. P. \& Chain, E. (1940). An enzyme from bacteria able to destroy penicillin. Nature, Lond. 146, 837.

Auhagen, E., Gloxhuber, C., Hecht, G., Knott, Th., Otten, H., Rauenbusch, E., Risse, K. H., Schmid, J., Scholtan, W. \& Walter, A.M. (1962). Ampicillin-Binotal ${ }^{(\mathbb{R})}$, ein Breitspektrum-Penicillin. Arzneimittel-Forsch. 12, 791.

AyıIFFe, G. A. J. (1963). Ampicillin inactivation and sensitivity of coliform bacilli. J. gen. Microbiol. 30, 339.

Barber, M. (1962). Resistance of Bacteria to the Penicillins, p. 86. Ciba Foundation Study Group, No. 13. London: J. and A. Churchill, Ltd.

Batchelor, F. R., Doyle, F. P., Nayler, J. H. C. \& Rolinson, G. N. (1959). Synthesis of penicillin: 6-aminopenicillanic acid in penicillin fermentations. Nature, Lond. 183, $25 \%$.

Bondi, A. \& Dietz, C. C. (1944). Production of penicillinase by bacteria. Proc. Soc. exp. Biol. N.Y. 56, 132.

Knox, R. \& Smith, J. T. (1962). Antibacterial activity, penicillinase stability, and inducing ability of different penicillins. J. gen. Microbiol. 28, 471.

Rolinson, G. N. \& Stevens, S. (1961). Microbiological studies on a broad-spectrum penicillin, 'Penbritin'. Brit. med. J. ii, 191.

Sмrтh, J. T. (1968). Penicillinase and ampicillin resistance in a strain of Escherichia coli. J. gen. Microbiol. 30, 299.

Stewart, G. T., Coles, H. M. T., Nixon, H. H. \& Holt, R. J. (1961). 'Penbritin': an oral penicillin with broad-spectrum activity. Brit. med. J. ii, 200.

Tномаs, R. (1961). Colorimetric detection of penicillins and cephalosporins on paper. Nature, Lond. 191, 1161.

Trafford, J. A. P., Maclaren, D. M., Lillicrap, D. A., Barnes, R. D. S., Houston, J. C. \& KNox, R. (1962). Ampicillin: a broad-spectrum penicillin. Lancet, i, 987. 